\title{
Eficacia de un programa de entrenamiento intradiálisis de fuerza-resistencia en combinación con electroestimulación neuromuscular: mejora en la capacidad funcional, fuerza, y calidad de vida
}

“Premio de investigación en enfermería nefrológica Janssen-Cilag 2010"

Gustavo Manuel Contreras Martos* - Miguel Delgado Rodríguez** - Juan Martínez Villar*** - Inmaculada Parra Mozas*** - Francisco Borrego Utiel**** - Pilar Segura Torres****

*Enfermero y Fisioterapeuta del Servicio de nefrología del Complejo Hospitalario de Jaén

${ }^{* *}$ Catedrático de Medicina Preventiva de la Universidad de Jaén

***Enfermero del Servicio de Nefrología del Complejo Hospitalario de Jaén

$* * * *$ Nefrólogo del Complejo Hospitalario de Jaén

\section{Resumen}

El propósito de este estudio fue determinar la eficacia de un programa de ejercicios de fuerza-resistencia durante la hemodiálisis, en la mejora de la fuerza muscular, calidad de vida y capacidad funcional para la realización de actividades de la vida diaria. Se realizó un estudio cuantitativo y experimental de tipo pretest y postest.

Se aplicó un programa de ejercicios de fuerzaresistencia en combinación con electroestimulación neuromuscular a 10 pacientes sometidos a hemodiálisis. Fueron tres ejercicios de sencilla ejecución adaptados a la posición en la que se realizaba la hemodiálisis.

Todos los pacientes mostraron una mejoría significativa en la fuerza, medida mediante test funcionales para la realización de las actividades de la vida diaria: caminar (6-MWT) y sentarse-levantarse (10-STS). Estos test fueron medidos antes y después

\section{Correspondencia:}

Gustavo Manuel Contreras Martos

c/ Fuente del Alamillo n³ portal 3,5²E. 23006. Jaén

E-mail:makogus@hotmail.com del programa de entrenamiento. También mostraron mejoría en la dimensión física de la calidad de vida medida mediante el cuestionario específico para el enfermo renal, el KDQOL-SFTM.

\section{PALABRAS CLAVE:}

- HEMODIÁLISIS

- CALIDAD DE VIDA

- ELECTROESTIMULACIÓN NEUROMUSCULAR

- CAPACIDAD FUNCIONAL

Efficacy of an intradialysis strength-stamina training programme in combination with neuromuscular electrostimulation: improvement in functional capacity, strength, and quality of life

\section{Abstract}

The purpose of this study was to determine the efficacy of a programme of strength-stamina exercises during haemodialysis, in improving muscular strength, quality of life and functional capacity to carry out 
everyday activities. A quantitative, experimental pre-test and post-test study was carried out.

A programme of strength-stamina exercises in combination with neuromuscular electrostimulation was applied to 10 patients undergoing haemodialysis. These were three simple exercises adapted to the position in which haemodialysis was carried out.

All the patients showed a significant improvement in strength, measured using functional tests to carry out everyday activities: walking (6-MWT) and sitto-stand tests (10-STS). These tests were measured before and after the training programme. They also showed an improvement in the physical dimension of the quality of life measured using the specific questionnaire for renal patients, KDQOL-SF ${ }^{\mathrm{TM}}$.

\section{KEY WORDS: \\ - HAEMODIALYSIS \\ - QUALITY OF LIFE \\ - NEUROMUSCULAR ELECTROSTIMULATION \\ - FUNCTIONAL CAPACITY}

\section{Introducción}

Existen datos considerables de que pacientes sometidos a hemodiálisis (HD), como grupo, tienen bajos niveles de actividad física y que los datos de hospitalización y de supervivencia son directamente proporcionales a la actividad física. A pesar de tan importante dato, los pacientes de diálisis tienen unos niveles bajos de actividad física diaria, si se compara con sujetos sanos como controles. Hasta el punto de que un paciente de 30 años sometido a HD tiene menos actividad física diaria que un individuo sano y sedentario de 70 años de edad ${ }^{1}$. Los pacientes con enfermedad renal crónica tienen un estado de forma física limitado y muchos factores que lo favorecen, como son anemia, disfunción cardíaca, anormalidades musculares, depresión, etc. Además, la debilidad muscular, la fatiga, calambres y mioclonias, que afectan predominantemente en los miembros inferiores, son algunos de los síntomas que sufren diariamente los pacientes en hemodiálisis, y que limita drásticamente su capacidad para trabajar o realizar actividades de ocio.
La atrofia muscular en los pacientes urémicos ha sido descrita como consecuencia de una neuropatía urémica causada por degeneración axonal primaria con desmielinización segmentaria, o como una miopatía urémica debida a una estructura y funcionalidad anormal de las fibras musculares. Una HD efectiva puede corregir la neuropatía urémica pero la miopatía persiste. Las fibras musculares de un paciente en HD tienen muchas alteraciones, debido posiblemente a las adaptaciones de esas fibras a un medio interno alterado. Esas alteraciones incluyen cambios en los capilares, enzimas, proteínas contráctiles, etc. La miopatía ocurre en un paciente urémico como consecuencia de unos niveles séricos de calcio elevados, azoemia, acidosis, bajos niveles de carnitina e hiperparatiroidismo primario 0 secundario. Un programa de ejercicios tiene efectos sobre la anemia, la función cardiovascular, la capacidad aeróbica, sobre la depresión y sobre la calidad de vida de estos pacientes ${ }^{2}$. El ejercicio físico se viene utilizando como herramienta terapéutica desde principios de los años $80^{3}$. Tres son las posibles modalidades: ejercicio en casa, ejercicio supervisado en días de no diálisis o ejercicio durante la sesión de HD4 . Esta última ha demostrado ser la modalidad más conveniente por el control de constantes del paciente durante el ejercicio y por favorecer la adhesión al programa.

El objetivo principal fue analizar los efectos de un programa de entrenamiento de fuerza-resistencia combinado con electroterapia en los pacientes durante la sesión de hemodiálisis, para comprobar las variaciones en la capacidad funcional (AVD: sentarse-levantarse y caminar) y calidad de vida (esfera física y mental). Se establecieron como hipótesis las siguientes: $\mathbf{1}^{\mathrm{a}}$ : Si a un paciente en hemodiálisis se le sometía a un programa de entrenamiento de fuerza-resistencia en miembros inferiores, entonces se produciría un aumento en la fuerza muscular y una mejora en la capacidad funcional (sentarse-levantarse y caminar). $2^{\mathrm{a}}$ : Si a un paciente en hemodiálisis se le sometía a un programa de entrenamiento de fuerza-resistencia en miembros inferiores, entonces se produciría una mejora en la calidad de vida.

\section{Metodología}

La metodología que se ha empleado es la de un estudio cuantitativo, experimental pre y postratamiento, en el que se ha cuantificado la eficacia de una intervención/ 
tratamiento en un grupo de pacientes sometidos a hemodiálisis. Se realizó una valoración antes y después de la intervención, empleando cada sujeto de la muestra como su propio control. Se llevó a cabo en un grupo determinado de 11 pacientes sometidos a hemodiálisis, a los cuales se les sometió a un programa de entrenamiento de fuerza-resistencia durante 5 semanas, en el Complejo Hospitalario de Jaén, en concreto en la Unidad de Crónicos del Servicio de Nefrología.

Se solicitó a los miembros del equipo sanitario que trataban a los pacientes (nefrólogos y personal de enfermería), un listado con los pacientes sometidos a hemodiálisis y que podían ser susceptibles de participar en el estudio. Los criterios de elegibilidad fueron los siguientes: edad comprendida entre 18 y 80 años, al menos 3 meses en tratamiento de hemodiálisis, encontrarse estable clínicamente. Esta condición de apto o no apto fue establecida por el equipo médico y de enfermería del servicio de Nefrología. Fueron considerados como criterios de exclusión los siguientes: ser portador de prótesis metálicas en la zona en la que se iban a colocar los electrodos de la electroterapia, realizar actividad física por cuenta propia y que no fueran las actividades de la vida diaria (AVD), empeoramiento del estado general, ser portador de una fístula arteriovenosa en miembros inferiores, en caso de presentar angina inestable al ejercicio o en reposo, patología vascular periférica importante, amputación de miembros inferiores sin prótesis, enfermedad vascular cerebral (ictus, isquemias transitorias), 0 alteraciones músculo-esqueléticas o respiratorias que empeoraran con el ejercicio. Fue excluido a todo paciente que en su historial clínico apareciera cualquier patología de carácter cardíaco, aunque el episodio hubiera sido posterior a 6 semanas y estuviera resuelto. Otros estudios similares admitieron a pacientes con patología coronaria, si ésta había ocurrido hacía más de tres meses de reiniciarse el estudio ${ }^{5}$.

El proyecto de esta investigación fue presentado ante la Comisión de Investigación del Complejo Hospitalario de Jaén en defensa ante la comisión el día 15 de mayo del 2009, con el visto bueno de la misma. Una vez aprobado el proyecto, se les facilitó a todos los participantes del mismo un consentimiento informado, en el que se detallaba toda la información que les interesaba conocer: los beneficios del ejercicio físico en la insuficiencia renal crónica avanzada, el tipo de ejercicios que iban a realizar, las pruebas de control pre y post que debían realizar y, si los hubiere, los riesgos derivados de la intervención. Una vez aplicados los criterios de inclusión y exclusión, el número de participantes fue muy reducido, ya que hay que tener en cuenta que la población de estudio disponible en ese momento tenía un estado general delicado. Hay que decir que en la Unidad de Crónicos se dializan los pacientes que deben ser vigilados más estrechamente por el personal sanitario, debido a su peor estado de salud. Algunos de estos pacientes suelen ser de más de 80 años y la gran mayoría tienen una comorbilidad importante, sobre todo patología cardíaca y vascular. La distribución de pacientes en la Unidad de Crónicos es en 3 turnos de enfermos. Por razones de factibilidad y de disponibilidad del equipo investigador no se eligieron a pacientes del tercer turno, tan solo de los dos primeros. Teniendo en cuenta esta salvedad, fueron un total de 11 los pacientes que cumplieron los criterios de inclusión. A las tres semanas de iniciada la intervención/tratamiento hubo un empeoramiento del estado general de uno de los pacientes, lo que le llevó al fallecimiento. Aunque se inició el estudio con 11 sujetos, lo finalizaron solo 10.

La intervención consistió en la realización de un programa de ejercicios combinado con electroterapia durante las dos primeras horas de tratamiento de hemodiálisis, en las cuales el paciente está más colaborador y dispuesto a realizar los ejercicios. El programa de ejercicios propuesto se prolongó por un espacio de 5 semanas y se realizó en las tres sesiones de diálisis semanales. Los ejercicios se centraron en potenciar el cuádriceps, músculo responsable de que la mayoría de las actividades para la vida diaria (AVD) se realicen de forma satisfactoria, otorgando al paciente mayor independencia. Los ejercicios se adaptaron a la posición que adoptaba el paciente durante la sesión de HD, es decir en sedestación. La sesión de entrenamiento comenzaba cuando el paciente se conectaba a la máquina de HD. Lo primero que se le realizaba era un ligero masaje estimulante del cuádriceps y del tríceps sural de 5 minutos como precalentamiento. A continuación se les realizaba los ejercicios del programa de entrenamiento que fueron los siguientes:

- Extensión de rodilla: extensiones de rodilla partiendo de la posición de $90^{\circ}$ a la posición de $0^{\circ}$ grados (la posición de sedestación que adoptaban para la HD). Para incrementar la dificultad se lastraron los tobillos con una carga siempre personalizada a la fuerza del 
paciente. Realizaron 3 series de 15 repeticiones, incrementándose la carga a medida que el paciente mejoraba su condición física. El objetivo fue que se mantuviera una constante de esfuerzo de entre 6 y 7 en la escala de Borg, por lo que se incrementaba la carga en los tobillos para conseguir esas puntuaciones en la valoración del esfuerzo percibido.

- Triple extensión de miembro inferior: extensión simultánea de cadera, rodilla y tobillo, teniendo como resistencia la oposición del fisioterapeuta en caso de pacientes con un balance muscular de 2-3 según escala de Daniels (Hislop H. et al. $)^{6}$. En pacientes con mejores balances se utilizaron bandas elásticas de Teraband ${ }^{\circledR}$ de resistencia media, fuerte o extrafuerte, según la fuerza del paciente. En algunos pacientes se llegó a utilizar dos bandas simultáneas, ya que con una sola no se conseguían puntuaciones de 6-7 en la escala de Borg. Se realizaron también 3 series de 15 repeticiones incrementando la resistencia progresivamente según las puntuaciones obtenidas en la escala de Borg.

- Contracción isométrica del cuádriceps: desde una posición inicial de extensión de rodilla, se le pedía al paciente que realizara una contracción mantenida del cuádriceps durante 6 segundos, sin realizar en ningún momento maniobra de Valsalva que elevara la tensión arterial por aumento de la presión intraabdominal. Realizaron un total de tres series de quince repeticiones.

- Exclusivamente para el tercer ejercicio se añadió la variante de electroestimulación neuromuscular (EENM). En el momento que el paciente notaba el paso de corriente éste realizaba una contracción isométrica. Las características del impulso eléctrico fueron las siguientes: fase de contracción de 3 segundos alternando con otra fase de relajación de 6 segundos de duración. Durante la fase de contracción el impulso tuvo una frecuencia de $50 \mathrm{~Hz}$ y una duración de 250 mseg, con una duración total de 3 segundos. La fase de relajación tuvo una duración del doble que la fase de contracción, para evitar que el músculo se fatigara y diera tiempo a que se recuperara; en total 9 seg. Los electrodos empleados fueron de $10 \times 5$ $\mathrm{cm}$ y se colocaron en ambos cuádriceps en sentido transversal, colocando el ánodo (polo positivo) en el punto motor que ofreciera una contracción más efectiva. El cátodo (polo negativo) se colocó en el tercio distal del muslo, en la inserción del cuádriceps. La intensidad de corriente se incrementó hasta conseguir contracción muscular tolerable y no dolorosa.

Se recogieron datos demográficos del paciente como edad, sexo, situación laboral, nivel de estudios, estado civil. Otras variables clínicas objeto de estudio fueron el tipo de acceso vascular, tiempo en hemodiálisis, peso, talla e IMC. Las variables recogidas relacionadas con la capacidad funcional y fuerza física fueron: TAS, TAD, Sat02, FC, Escala de Borg en test 6-MWT y 10-STS, distancia recorrida en el 6-MWT, tiempo empleado en el 10-STS, balance muscular pierna drcha e izqda mediante la escala de Daniels que puntúa la fuerza muscular de 0 a 5 (Hislop $\mathrm{H}$, et al. $)^{6}$ y la carga levantada en $1^{\circ}$ ejercicio del entrenamiento. La calidad de vida se recogió mediante el test $K D Q 0 L-S F$, valorándose la dimensión física y mental.

Las pruebas funcionales encaminadas a evaluar la condición física de los pacientes fueron el test de 6 minutos marcha $(6 \mathrm{MWT})^{7}$ y la prueba de sentado a de pie y de nuevo a sentado ('sit to stand to sit' o STS) de 10 repeticiones $(\mathrm{STS} 10)^{8}$.

- El test de $6 \mathbf{M W T}^{7}$ se realizó previamente a la $3^{\mathrm{a}}$ sesión semanal de hemodiálisis, en el pasillo de la unidad. En el momento previo a la realización de la prueba se registró la frecuencia cardiaca basal, la tensión arterial en el brazo sin FAVI y la sat $\mathrm{O}_{2}$. A continuación se le indicó al paciente que, durante 6 minutos, recorriera el mayor número de veces posible la distancia predeterminada y medida de antemano, en este caso el pasillo de la unidad. En este pasillo se midió una distancia de 25 metros, se hicieron marcas en el suelo con cinta tanto en los extremos como cada 3 metros. El investigador principal se colocaba en uno de los extremos del circuito y otro colaborador en el otro. A ambos lados se colocaron sendas sillas por si el paciente se cansaba durante la prueba. Se pusieron otras dos sillas más en puntos intermedios del circuito. Las instrucciones que se le dieron al paciente fueron la de que caminara tan rápidamente como pudiera, sin correr pero a un ritmo que notase que no hubiese podido caminar más deprisa. Si el paciente precisaba de ayudas para la marcha, bien instrumentales o por otra persona, se les permitió utilizarlas en la prueba, incluso si necesitaba descansar podría hacerlo. En concreto uno de los sujetos del estudió que precisaba de bastón para caminar, 
se le permitió utilizarlo para la prueba. Se registró la distancia recorrida y se le pidió al paciente que describiera en la escala de esfuerzo percibido- EEP (escala de Borg) cuál había sido el grado de dificultad de la prueba. En todo momento iba monitorizado con pulsómetro y pulsioxímetro portátil.

- Pruebas del STS-108: tal y como describen Csüka y McCarty consistía en medir los segundos que necesitaba el paciente para, desde una posición de sentado, levantarse y volver a sentarse 10 veces consecutivas lo más rápidamente posible, con los brazos pegados al pecho. La silla empleada fue una estándar de $43 \mathrm{~cm}$ con el respaldo pegado a la pared. Se le permitía un intento de prueba. Tras realizar las 10 repeticiones se registraba el tiempo empleado, así como el grado de dificultad en la EEP de Borg. En esta prueba el paciente podía descansar todas las veces que fuera necesario, sin que llegase a ser una actividad extenuante. Se tomó la frecuencia cardiaca basal, tensión arterial y sat $\mathrm{O}_{2}$ antes y después de la prueba. También iba monitorizado.

- Balance muscular según la escala de Daniels. El mismo día de la realización del test de STS-10, una vez sentado el paciente en la silla, y antes de la realización de dicho test, el investigador principal sometió al paciente a un test de balance muscular para valorar la fuerza muscular para la extensión de rodilla, en el que interviene de forma casi exclusiva el cuadriceps. El investigador se colocó frente al sujeto, hizo una toma con la mano no dominante en el muslo por encima de la rodilla, y la mano dominante en el tercio distal de la pierna. Imprimió una fuerza hacia el suelo y le pidió al paciente que impidiera que la pierna se flexionara. El investigador puntuó según la resistencia que ofreció a la fuerza.

Para la objetivación de la calidad de vida se utilizó el cuestionario KDQOL-SFTM antes y después del programa de ejercicios. A todo paciente con la capacidad suficiente como para que pudiera rellenarlo él en casa, se le entregó para que lo contestara de forma autónoma, permitiendo que hiciera preguntas y planteara dudas. A aquellos que por alguna limitación no pudieron rellenarlo, se le ayudó en la sesión de HD, en las primeras 2 horas. Del total de pacientes hubo 5 a los que hubo que ayudarles a rellenar el cuestionario. Se realizó en el día previo o en el mismo día de comienzo del plan de entrenamiento. Sus items dieron puntuaciones sobre diferentes subescalas, puntuando de 0 (peor nivel de salud) a 100 (mejor nivel de salud $)^{9}$.

Los datos fueron analizados mediante el programa de análisis estadístico SPSS v.15. El cuestionario de calidad de vida se analizó con el programa KDQOLSF TM Version 1.3 Scoring Program (v 3.0). Para cada variable se han hallado los valores descriptivos de tendencia central y de dispersión más habituales (media, desviación estándar) que se presentan en sus correspondientes tablas. En cuanto a la estadística inferencial, el procedimiento seguido para el análisis y realización de los contrastes de hipótesis fue el siguiente. La muestra elegida no cumplía los criterios para emplear estadísticos paramétricos, ya que tenía un escaso tamaño muestral $(\mathrm{N}=10)$ y además su selección no había seguido criterios de aleatorización estricta. Por este motivo se han empleado pruebas no paramétricas para su análisis, las cuales no presuponen una distribución de probabilidad para los datos. En concreto, el estadístico utilizado ha sido la prueba de Wilcoxon para contrastar datos pareados, ya que el análisis de este estudio se basa en la comparación de los datos de cada una de las variables antes y después del programa de entrenamiento de fuerza. Por tanto, éste será el estadístico de elección. Se ha empleado el límite de $p<0,05$ para considerar un resultado como significativo.

\section{Resultados}

A continuación se exponen los datos de las variables del estudio en tablas, expresados con una medida de tendencia central (media) y otra de dispersión (desviación estándar).

Los datos clínicos generales de la muestra vienen expresados en la Tabla 1.

\begin{tabular}{|ll}
\hline Edad (años) & $66,6 \pm 11,5$ \\
\hline Tiempo en hd (meses) & $38 \pm 13$ \\
\hline Peso (kg) & $72,2 \pm 15,4$ \\
\hline Talla (cm) & $162 \pm 9,3$ \\
\hline IMC (kg/ $\left.\mathrm{cm}^{2}\right)$ & $26.9 \pm 5,5$ \\
\hline $\begin{array}{l}\text { Acceso vascular (FAVI/ Catéter Venoso } \\
\text { Permanente (\%)) }\end{array}$ & $60 / 40$ \\
\hline
\end{tabular}

Tabla 1: Datos clínicos generales 
En la Tabla 2 aparecen los resultados obtenidos en las variables clínicas relacionadas con el ejercicio físico.

\begin{tabular}{|l|l|l|l|}
\hline & Basal & Final & $P$ \\
\hline TAS $(\mathrm{mmHg})$ & $157,8 \pm 7,3$ & $151,7 \pm 13,2$ & 0,113 \\
\hline TAD $(\mathrm{mmHg})$ & $77,2 \pm 10,8$ & $80,9 \pm 8,7$ & 0,959 \\
\hline FC (Ipm) & $\begin{array}{l}113,5 \pm \\
22,7\end{array}$ & $111 \pm 15,9$ & 0,878 \\
\hline \begin{tabular}{l} 
Sat02 (\%) \\
\hline $\begin{array}{l}\text { 6-MWT-Escala de borg } \\
\text { (0-10) }\end{array}$
\end{tabular} & $65,2 \pm 1,5$ & $96,8 \pm 0,9$ & 0,016 \\
\hline $\begin{array}{l}\text { 10-STS-Escala de borg } \\
\text { (0-10) }\end{array}$ & $6,6 \pm 0,6$ & $7 \pm 0,2$ & 0,064 \\
\hline
\end{tabular}

Tabla 2: Variables relacionadas con el esfuerzo físico

En la capacidad funcion al es donde se han conseguido los mejores resultados, ya que se han encontrado diferencias estadísticamente muy significativas $(p<0,05)$ entre las pruebas de capacidad y fuerza realizadas pretest, y las conseguidas en las mismas pruebas postest. Todos los sujetos experimentaron una mejoría en mayor o menor medida. Los datos quedan recogidos en la Tabla 3:

\begin{tabular}{|l|l|l|l|}
\hline & Basal & Final & $P$ \\
\hline 6-MWT (metros) & $428 \pm 106$ & $492 \pm 100$ & 0,005 \\
\hline 10-STS (seg) & $35,3 \pm 20,3$ & $26,4 \pm 6,43$ & 0,005 \\
\hline $\begin{array}{l}\text { Balance muscular } \\
\text { drcho (0-5) }\end{array}$ & $4,1 \pm 0,5$ & $4,7 \pm 0,3$ & 0,014 \\
\hline $\begin{array}{l}\text { Balance muscular } \\
\text { izqdo (0-5) }\end{array}$ & $4,2 \pm 0,6$ & $4,9 \pm 0,3$ & 0,014 \\
\hline Carga levantada (kg) & $2,7 \pm 1,4$ & $4,1 \pm 1,3$ & 0,005 \\
\hline
\end{tabular}

Tabla 3: Variables relacionadas con la capacidad funcional

Los resultados en el apartado de calidad de vida, una vez analizado el cuestionario según el programa KDQOLSFTM Version 1.3 Scoring Program (v 3.0), se exponen en la Tabla 4.

\begin{tabular}{|l|l|l|l|}
\hline & Basal & Final & $P$ \\
\hline KDQOL físico (0-100) & $38,4 \pm 12,9$ & $45,3 \pm 13,1$ & 0,022 \\
\hline KDQOL mental (0-100) & $46,3 \pm 12,6$ & $49,5 \pm 13,1$ & 0,093 \\
\hline
\end{tabular}

Tabla 4: Calidad de vida

\section{Discusión}

Se han obtenido resultados muy positivos en las pruebas indirectas realizadas para medir la fuerza y la capacidad funcional de los sujetos para la realización de actividades para la vida diaria (AVD), lo que sugiere que el programa de fuerza-resistencia durante la HD produjo una mejora muscular. Este hallazgo coincide con las observaciones de estudios previos, que demostraron mejoras significativas en el test 10-STS, tanto con la aplicación de un programa de ejercicio de fuerza-resistencia muscular en los días de no $\mathrm{HD}^{(10)}$, como con la aplicación de ejercicio combinado aeróbico y de resistencia durante la $\mathrm{HD}^{11}$.

La mejora en la prueba de 6-MWT se ha visto en otros estudios que han aplicado programas exclusivos de fuerza en sujetos en prediálisis, aunque la primera vez que un programa de ejercicios durante el hemodiálisis en España demostró que aumentaba de forma significativa el resultado de esta prueba, fue el de Segura et $\mathrm{al}^{5}$. Este estudio incluyó a 16 pacientes con una media edad de 54 años a los cuales dividió en dos grupos: el de intervención con 8 pacientes y el de control, también con 8 (el grupo control fue formado por aquellos pacientes que se negaron a realizar el programa entrenamientos). Aunque los resultados de las pruebas fueron similares a los del presente estudio, hay que destacar que a pesar de que la media de la del estudio de Segura et $\mathrm{al}^{5}$ era de 54,9 frente a los 66 años de media de nuestro estudio, y de que su programa de entrenamiento fue de 6 meses frente al nuestro de tan solo 5 semanas, nuestros pacientes fueron capaces de caminar más distancia que ellos $(399 \pm 39,5 \mathrm{~m}$ frente a 471,7 $\pm 70,6 \mathrm{~m}$ para Segura et al; $428 \pm 106 \mathrm{~m}$ frente a $492 \pm 100 \mathrm{~m}$ de nuestro estudio). Sin embargo, los pacientes de nuestro estudio fueron más lentos en la prueba de 10 -STS $(22,5 \pm 4,77$ seg. frente a 17,71 $\pm 1,79$ seg. para Segura et al; $35,3 \pm 20,3$ seg. frente a $26,4 \pm 6,43$ para nuestro estudio). Una diferencia notable de nuestro estudio con el de Segura et. al ha sido el empleo de electroterapia en la realización de ejercicios isométricos de cuádriceps. También se han obtenido resultados significativos en el balance muscular tanto de la pierna derecha como de la pierna izquierda, esto significa que los pacientes ganaron fuerza en la musculatura extensora de la rodilla cuando ésta fue valorada 6 . En cuanto a la carga levantada por los pacientes en el primer ejercicio del programa de 
entrenamientos, ya desde los primeros días se empezó a notar una mejoría espectacular, ya que con la misma intensidad esfuerzo (Borg) los pacientes eran capaces de levantar más carga $(2,7 \pm 1,4$ inicial frente a los $4,1 \pm 1,3$ al final del programa

En cuanto a calidad de vida hay que ser muy cautos a la hora de interpretar los resultados, ya que en la calidad de vida hay muchos factores que intervienen ${ }^{12}$. No obstante, en nuestro estudio se han obtenido resultados significativos para la dimensión física de la calidad de vida según el KDQOL-SF. Lo que está claro es que existe una asociación entre mayor facilidad para la realización de actividades para la vida diaria de forma independiente, y una mayor calidad de vida en la esfera física y mental ${ }^{9}$. A pesar de esto, nos sorprende que en nuestro estudio no haya habido diferencias significativas en la esfera mental de la calidad de vida como en realidad esperábamos, aunque este hecho se puede deber a que la calidad de vida está muy influenciada por la edad y el estado de salud, y dado que nuestra media de edad es elevada y la comorbilidad de nuestros pacientes también lo es, es por lo que la calidad de vida en su esfera mental es baja y no ha respondido a como nosotros esperamos.

\section{Conclusiones}

El objetivo fundamental de esta investigación era averiguar si realizando un programa de ejercicios de fuerza-resistencia se producían cambios en la fuerza y la capacidad funcional para realizar actividades de la vida diaria como caminar y levantarse de una silla, así como en la calidad de vida. Nuestros resultados han confirmado este objetivo principal cumpliéndose la hipótesis 1 de que aumentaría la capacidad funcional para realizar actividad física relacionada con la vida diaria. También se ha cumplido la hipótesis 2 que decía que mejoraría la calidad de vida de los pacientes aunque hay que decir que esta hipótesis se ha cumplido sólo a medias, ya que ha mejorado sólo la calidad de vida en la esfera física y no en la mental. Como conclusión, un programa de trabajo de fuerza resistencia intradiálisis aumentó la capacidad funcional y mejoró el componente físico de la calidad de vida de los pacientes, por lo que se justificaría la incorporación a los tratamientos habituales de diálisis una pauta de ejercicios totalmente personalizada.
Recibido: Abril 2011

Revisado: Abril 2011

Modificado: Mayo 2011

Aceptado: Mayo 2011

\section{Bibliografía}

1. Ikizler Alp T, Himmelfarb J. Muscle casting in kidney disease: let's get physical. J Am Soc Nephrol. 2006; 17: 2097-2098.

2. Kouidi $E$, Iacovides $A$, Iordanis $P$ et al. Exercise renal rehabilitation program (ERRP): psycosocial effects. Nephron. 1997; 77: 152-158.

3. Painter $\mathrm{PL}$, Nelson-Worel JN, Hill MM, Thornbery DR, Shelp WR, Harrington AR, et al. Effects of exercise training during hemodialysis. Nephron. 1986; 43: 87-92.

4. Kouidi EJ. Central and Peripheral adaptations to physical training in patients with end-stage renal disease. Sports Med. 2001; 31(9): 651-65.

5. Segura E, Rodilla-Alama V, Lisón JF. Fisioterapia durante la hemodiálisis: resultados de un programa de fuerza-resistencia. Nefrología. 2008; 28 (1): 67-72.

6. Hislop H. Daniels \& Worthinghan. Técnicas de balance muscular. $7^{a}$ ed.: Elsevier; 2003. p. 2.

7. ATS Statement. Guidelines for the Six-Minute Walk Test. Am. J. Respir Crit. Care Med. 2002; 166: 111-117.

8. Baldini $M$, Bernal $A$, Jiménez $R$ y Garatachea $N$. Valoración de la condición física en ancianos. Revista Digital-Buenos Aires [serie en Internet]. 2006 [citado Dic 2006]; 103. Disponible en: http://www. efdeportes.com/efdl03/condic.htm.

9. Hays RD, Kallich JD, Mapes DL, Coons SJ, Armin N, y Carter WB. Kidney Disease Quality of Life Short Form (KDQOL-SFTM), Version 1.3: A manual for Use and Scoring. 1995; Santa Mónica, CA: RAND, P-7994. 
10. Headley S., Germain M., Mailloux P. et al. Resistance Training Improves Strength and Functional Measures in Patients With End-Stage Renal Disease. Am J Kidney Dis 2002; 40 (2): 355-364.

11.Painter P, Moore G, Carlson L, Paul S, Myll J, Phillips $W$, et al. Effects of exercise training plus normalization of hematocrit on exercise capacity and health-related quality of life. Am J Kidney Dis. 2002; 39(2): 257-65.

12. Magaz Lago A. Efectos psicológicos de la insuficiencia renal: diferencias en función de los tratamientos médicos [tesis doctoral]. Bilbao: Universidad de Deusto; 2003. 